\title{
Dental Management of Pediatric Patients with Bronchial Asthma
}

\author{
Karishma Chhabra ${ }^{1}$, Shveta Sood ${ }^{2}$, Naresh Sharma ${ }^{3}$, Akshara Singh $^{4}$, Sankalp Nigam ${ }^{5}$
}

\begin{abstract}
Asthma is a disease marked by recurrent dyspnea caused by spasmodic bronchoconstriction. Bronchial asthma is a serious global problem that has steadily increased in prevalence during the past two decades. Oral manifestations of pediatric patients suffering from bronchial asthma are an increased upper anterior and total anterior facial height, higher palatal vaults, greater overjets, and a higher prevalence of posterior crossbites. Dental treatment of such patients requires the role of a pedodontist. Behavior management techniques, conscious sedation techniques along with medical management, and comprehensive dental treatment by a pediatric dentist may prove beneficial to such patients.

Keywords: Asthma, Pediatric dentistry, Pediatric patient.

International Journal of Clinical Pediatric Dentistry (2021): 10.5005/jp-journals-10005-2024
\end{abstract}

\section{INTRODUCTION}

As defined by World Health Organization, asthma is a disease characterized by "recurrent attacks of breathlessness and wheezing, which vary in severity and frequency from person to person. In an individual, they may occur from hour to hour and day-to-day". Asthma is a disease marked by recurrent dyspnea caused by spasmodic bronchoconstriction. ${ }^{1}$ Bronchial asthma is a serious problem that has steadily increased in prevalence during the past two decades. A history of asthma is reported commonly by adult dental patients and may be more prevalent in children. An asthma episode should be considered a medical emergency and must be treated promptly by inhalation of a bronchodilating agent. A history of asthma in the dental patient should alert the dentist to implement strategies that may prevent an acute attack and be prepared to manage this potentially life-threatening medical emergency appropriately. Asthmatic patients need special attention during dental surgery to avoid wheezing and bronchospasm. ${ }^{1}$ Classically, the asthmatic patient has hypersensitivity of the entire tracheobronchial tree to pulmonary irritants and allergens. Exacerbations of asthma caused by physical and emotional stress may also occur. Asthma is usually classified by combined clinical and etiological factors. Asthma is a disease marked by recurrent dyspnea caused by spasmodic bronchoconstriction. Classically, the asthmatic patient has hypersensitivity of the entire tracheobronchial tree to pulmonary irritants and allergens. ${ }^{2}$ Exacerbations of asthma caused by physical and emotional stress may also occur. Asthma is usually classified by combined clinical and etiological factors into one of two principal forms, extrinsic and intrinsic asthma. ${ }^{3}$ Extrinsic or allergic asthma is the type most commonly seen in children and young adults. Acute attacks are often related to exposure to specific allergens. ${ }^{4}$ Patients may have family histories of asthma and other allergic phenomena, such as seasonal rhinitis, foodinduced eczema, and urticaria. These patients are often symptomfree between attacks, respond well to medical therapy, and have good long-term prognoses. Intrinsic, or infectious asthma, is most commonly seen in patients older than 35 years. Allergens are, for the most part, not the etiological agents; and often infection is a causative factor. In addition, chronic cough and increased sputum production tend to develop in patients between acute attacks,

\footnotetext{
${ }^{1-5}$ Department of Pedodontics and Preventive Dentistry, Manav Rachna Dental College, Faridabad, Haryana, India

Corresponding Author: Karishma Chhabra, Department of Pedodontics and Preventive Dentistry, Manav Rachna Dental College, Faridabad, Haryana, India, Phone: +91 9871175755, e-mail: karishma. chabra18@gmail.com

How to cite this article: Chhabra K, Sood S, Sharma N, et al. Dental Management of Pediatric Patients with Bronchial Asthma. Int J Clin Pediatr Dent 2021;14(5):715-718.

Source of support: Nil

Conflict of interest: None
}

which may lead to chronic obstructive pulmonary disease. In many such patients, nasal polyps will develop and cause occasional bouts of severe epistaxis. ${ }^{5}$ Approximately $10 \%$ of intrinsic asthmatic patients have salicylate intolerance. A detailed medical history is often necessary to distinguish the intrinsic from the extrinsic form of asthma. "Acute attacks of both entities may produce similar clinical pictures. Some patients cannot be unequivocally classified as having one of the two principal forms because of the complexity and variability in the clinical course of asthma. ${ }^{6}$

\section{Medical Management of Asthmatic Patients}

Acute and chronic asthma are classified into mild, moderate, and severe according to the severity and frequency of occurrence of the signs and symptoms and according to the degree of airway obstruction as measured with the pulmonary function test. The medical management of childhood asthma is determined according to these criteria. ${ }^{7}$ However, the severity of either acute or chronic asthma may vary within the same patient over time, and therefore adjustments in treatment frequently are necessary."

The goals of asthma therapy according to National Heart, Lung, and Blood Institute guidelines. ${ }^{8}$

- Maintenance of normal activity levels.

- Maintenance of normal pulmonary function. 
- Prevention of chronic symptoms.

- Prevention of recurrent exacerbations of asthma.

- Avoid adverse effects caused by asthma medication. Chronic childhood asthma may be treated by:

- Anti-inflammatory agents.

- Bronchodilators.

- Inhaled beta 2 receptor agonist bronchodilators, e.g., albuterol.

- Terbutaline sulfate can be used for treating children suffering from mild asthma.

Anti-inflammatory agents, e.g., cromolyn sodium and nedocromil sodium are used for treating moderate asthma. They prevent mast cell release of mediators. ${ }^{9}$ Prophylactic treatment with cromolyn and nedocromil may prevent allergeninduced asthmatic attacks and may decrease airway reactivity. But they are not useful for acute asthma responses. Oral theophylline may also be used for the treatment of moderate asthma. It has a bronchodilator and some anti-inflammatory properties. Inhalational corticosteroids are the most effective: anti-inflammatory agents. They may be used in children having moderate to severe asthma. Inhalational steroids are efficient for the treatment of chronic asthma and are convenient as they can be consumed twice daily.

\section{Oral Manifestations ${ }^{10}$}

- Increased susceptibility to dental caries.

- Dentofacial deformities.

- Side effects of prolonged use of steroids.

\section{Increased Susceptibility to Dental Caries}

According to a 1993 retrospective study performed in Sweden, it was found out that children with moderate to severe asthma have an increased prevalence of caries.

Another report suggested that the medications reduce the secretion of the whole saliva by $20 \%$ and parotid saliva by $35 \%$, and are also linked with a surge in the number of lactobacilli. These factors result in increased caries susceptibility. Because of this risk, special attention should be given to the prevention of caries in asthmatic children.

\section{Dentofacial Deformities}

Increased prevalence of dentofacial deformities is seen, e.g., increased upper anterior and total anterior facial height, higher palatal vaults, greater overjets, higher prevalence of posterior crossbites. $^{11}$

The cause behind the increased prevalence of dentofacial deformities is suggested to be respiratory disorders, e.g., chronic rhinitis, mouth breathing. ${ }^{1}$

\section{Side Effects of Prolonged Use of Steroids}

Inhalational steroids may have prominent adverse effects such as

- Adrenal suppression.

- Growth impairment.

- Throat irritation.

- Dysphonia.

- Dryness of the mouth.

- Oropharyngeal candidiasis.

\section{Dental Management}

The safe dental intervention of children having asthma is dependent on the pulmonary status of the child. Treatment of patients having asthma requires the dentist to consider the following:

- The frequency of asthmatic attacks.

- The type of medications used chronically and during acute attacks.

- The length of time since the child was last seen emergently with acute asthma.

Physical examination may include auscultation of the lungs, observing the rate and depth of respiration, use of accessory muscles for respiration, shortness of breath, and coughing. ${ }^{12}$

Dental procedures may be performed in the clinic setting for asymptomatic asthmatic patients. A wheezing or poorly controlled patient should be recalled for a later appointment. If the patient is currently using an inhaler bronchodilator, it needs to be carried along for the dentist's appointment. Dental anxiety may trigger an asthmatic attack in the dental office. So, the dental staff need to be relaxed and should make use of various techniques to alleviate anxiety in the child.

If there is a need for conscious sedation, hydroxyzine and benzodiazepines may be used. Dental treatment in pediatric patients with asthma should not include barbiturates and narcotics (mainly morphine and meperidine) as they may cause bronchospasm.

Nitrous oxide may be used for the management of pediatric patients with asthma because of its analgesic and anxiolytic properties and supplemental oxygen. It may be used in pediatric patients with mild to moderate asthma. Nitrous oxide may irritate the airway, it is contraindicated in pediatric patients with severe asthma.

IV sedation may be given in pediatric patients with asthma but with extra precaution because asthmatics have limited control over their respiratory airways. Ketamine is a safe drug for asthmatic patients. It is a dissociative anesthetic with sedative effects, analgesic effects, and bronchodilation. Ketamine should not be used in patients with cardiovascular or hypertensive heart disease as it causes activation of the sympathetic system. Before the treatment of patients with moderate to severe asthma, standard monitors (pulse oximetry, end-tidal $\mathrm{SAGO}_{2}, \mathrm{EKG}$, and blood pressure cuff) and intubation equipment should be used. Asthmatic patients with asthma where abrupt and severe episodes of airway obstruction occur should be treated in a hospital setup.

Before the commencement of dental treatment of pediatric patients in children with asthma, informed consent should positively be obtained from the concerned physician.

Acetaminophen may be used for the treatment of pediatric patients with asthma. As $4 \%$ of patients with asthma are allergic to aspirin and other non-steroidal anti-inflammatory agents.

Patients consuming theophylline preparations should not be prescribed erythromycin, as it interferes with the metabolism of theophylline and leads to its toxicity in the blood. ${ }^{13}$

Historically, the combination of local anesthetics and vasoconstrictor has been avoided for dental intervention in pediatric asthmatic patients due to the presence of sodium metabisulfite, a highly allergenic agent. Despite the warning, local anesthetics with vasoconstrictors have been used safely used. 
Another viewpoint suggests local anesthetics with vasoconstrictors should be administered cautiously since they may add to the effects of beta 2-agonists, leading to palpitations, increased blood pressure, and arrhythmias. ${ }^{14}$

Children with asthma who take systemic glucocorticoids (GC) may develop adrenal insufficiency during major dental procedures or general anesthesia. Compared with children without asthma, their risk of postoperative anesthesia-related complications is also higher (up to three times). Children with asthma who receive systemic maintenance GC (daily or every other day) develop adrenal suppression and must be supplemented by doubling the patient's usual daily dose on the day of dental surgery. ${ }^{15}$ Children are at risk of adrenal insufficiency due to major dental surgery or general anesthesia, including patients who received 4 or more short-term ( 45 days/course) or 1,014 consecutive days of systemic GC treatment for acute asthma in the previous year, and received within 30 days. Patients treated with systemic GC.

These children may need to receive pressure replacement steroids (hydrocortisone $60 \mathrm{mg} \mathrm{m}^{2} /$ dose) 68 hours before surgery and 1 hour before surgery, although this is not universally accepted by all authors. Children with asthma do not fall into these categories. Children with severe asthma need to be evaluated for anesthesia to avoid the risk of perioperative and postoperative complications. A patient history review, physical examination, and pulmonary function assessment (chest X-ray and maximum expiratory flow or spirometry) in selected patients may be required to identify patients who need to adjust their asthma treatment before dental treatment. Pulmonary function tests can identify some patients who are asymptomatic but have an obvious obstruction and FEV1 (forced expiratory volume in 1 second, a common indicator of airway obstruction) is below average. Dental treatment should be postponed to asthma control in patients with poorly controlled asthma and nocturnal wheezing, frequent severe seizures, bronchospasm induced by uncontrolled exercise.

Take the following steps to respond to an acute asthma attack in a dental office: ${ }^{.16}$

- Stop dental treatment and let the patient sit or lie down in a comfortable position.

- Keep the airway open and use an inhaler or nebulizer.

- Oxygen should be administered through a mask nasal mask or 1 cannula.

If there is no improvement, the patient's condition deteriorates, and epinephrine is injected subcutaneously (1:1,000 solution, $0.01 \mathrm{mg} / \mathrm{kg}$ body weight, the maximum dose is $0.3 \mathrm{mg}$ ), medical assistance, it is very important to continue treatment and maintain a satisfactory oxygen saturation until the patient no longer wheezes or until the patient can be taken to the emergency room using medical transportation. The incident should be recorded in detail and reported to the child's general practitioner. And lastly, in the dental management of foreign body inhalation, the rapid intervention of the dentist is very important. The basic method in this situation is adequate prevention. ${ }^{17,18}$

The dentist should be able to recognize and treat the disease immediately, thus contributing to the overall treatment of these patients.

\section{Foreign Body Aspiration ${ }^{15}$}

Many dental materials and components are small in size and can be difficult to handle properly when in contact with saliva.
If the patient lies on his back or is half-lifted, these objects may be swallowed or sucked into the oropharynx. Depending on the size, shape, and flexibility of the object, the intake may be small or it may be fatal.

In this case, prevention is clearly the best approach, although appropriate and prompt action in the event of accidental inhalation is essential to ensure patient safety. If a foreign body is inhaled into the oropharynx, the patient should straighten up and ask to cough vigorously. Ensuring that the airway remains open is a top priority. Accompanied by respiratory dysfunction, clearly identifiable symptoms such as suffocation, inhalation wheezing, and dyspnea, and rapid development of auxiliary muscle support. ${ }^{3}$

If a severe cough does not help, the Heimlich method should be used: we hold the patient from behind with our arms while standing. In this position, we apply pressure with a closed fist and cover the fist with the other hand. Put the fist on the top of the abdomen with the thumb, and then we press hard in the middle of the abdomen, just below the chest. If this operation proves to be ineffective, the patient must be transferred to the nearest emergency room as soon as possible. While waiting for the patient to be transferred, the dentist should take important measures, including penetrating the airway through cricothyrotomy if necessary. If the airway is not affected, the swallowed object should be retracted to give the patient peace of mind. If the item cannot be found, the patient should be explained to the patient, and appropriate chest and abdomen X-rays and clinical examinations in the hospital will help determine the location of the item. ${ }^{16}$

\section{Conclusion}

Dental treatment of pediatric patients with asthma can be efficiently carried out in presence of a well-equipped dental team and parental support. A pediatric dentist plays a major role in alleviating the anxiety of the child, maintaining a positive rapport with the child, and hence, making the dental treatment a comfortable and hasslefree experience for pediatric patients with asthma.

\section{References}

1. Geist ET, Diaz JH. Management of the asthmatic patient undergoing dental surgery. J Am Dent Assoc 1982;105(1):65-69. DOI: 10.14219/ jada.archive.1982.0060.

2. Zhu JF, Hidalgo HA, Holmgreen WC, et al. Dental management of children with asthma. Pediat Dentis 1996;18(5):363-370.

3. Claramunt Lozano A, Sarrión Pérez MG, Gavaldá, et al. Dental considerations in patients with respiratory problems. J Clin Exp Dent 2011;3(3):222-227. DOI: 10.4317/jced.3.e222.

4. Harrington N, Prado N, Barry S. Dental treatment in children with asthma - a review. Br Dent J 2016;220(6):299-302. DOI: 10.1038/ sj.bdj.2016.220.

5. Asthma UK, Asthma facts and statistics. Available online at https:// www.asthma.org.uk/asthma-facts-and-statistics (accessed November 2015).

6. Global initiative for asthma. Global burden of asthma. Available online at http://www.ginasthma.org/Global-Burden-of-Asthma (accessed November 2015).

7. Rajan JP, Wineinger NE, Stevenson DD, et al. Prevalence of aspirinexacerbated respiratory disease among asthmatic patients: A metaanalysis of the literature. J Allergy Clin Immunol 2015;135(3):676-681. DOI: 10.1016/j.jaci.2014.08.020.

8. Maupome G, Shulman J, Medina-Solis C, et al. Is there a relationship between asthma and dental caries? J Am Dent Assoc 2010;141(9):10611074. DOI: 10.14219/jada.archive.2010.0335. 
9. Steinbacher DM, Glick M. The dental patient with asthma: an update and oral health considerations. J Am Dent Assoc 2001;132(9):12291239. DOI: 10.14219/jada.archive.2001.0365.

10. Center for Disease Control and Prevention website. Retrieved August 21, 2017 from https://www.cdc.gov/asthma/default.htm.

11. Roland JN, Bhalla RK, Earis J. The local side effects of inhaled corticosteroids: current understanding and review of the literature. Chest 2004;126(1):213-219. DOI: 10.1378/chest.126.1.213.

12. Kurt $\mathrm{E}$, Yildirim $\mathrm{H}$, Kiraz $\mathrm{N}$, et al. Oropharyngeal candidiasis with dry-powdered fluticasone propionate: $500 \mathrm{microg} /$ day versus 200 microg/day. Allergol Immunopathol 2008;36(1):17-20. DOI: $10.1157 / 13115666$.

13. Fukushima C, Matsuse H, Saeki S, et al. Salivary IgA and oral candidiasis in asthmatic patients treated with inhaled corticosteroid. J Asthma 2005;42(7):601-604. DOI: 10.1080/02770900500216259.
14. Knight $\mathrm{L}$, Fletcher J. Growth of Candida albicans in saliva: stimulation by glucose associated with antibiotics, corticosteroids, and diabetes mellitus. J Infect Dis 1971;123(4):371-377. DOI: 10.1093/ infdis/123.4.371.

15. Metha A, Sequeira PS, Sahoo RC, et al. Is bronchial asthma a risk factor for gingival diseases? A control study. N Y State Dent J 2009;75(1):44-46.

16. Stensson M, Wendt L-K, Koch G, et al. Oral health in young adults with long term, controlled asthma. Acta Odontol Scand 2011;69(3):158164. DOI: 10.3109/00016357.2010.547516.

17. Guggenheimer J, Moore PA. The patient with asthma: implications for dental practice. Compend Contin Educ Dent (Jamesburg, NJ: 1995) 2009;30(4):200-202.

18. Paganini M, Dezan CC, Bichaco TR, etal. Dental caries status and salivary properties of asthmatic children and adolescents. Int J Paediatr Dent 2011;21(3):185-191. DOI: 10.1111/j.1365-263X.2010.01109.x. 